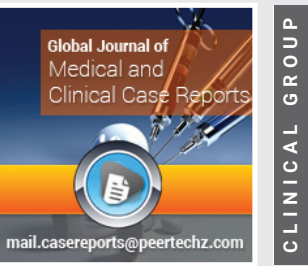

\title{
Charcot Neuroarthropathy in a Diabetic Patient. The Need for a Multidisciplinary Interventions
}

Received: 05 March, 2020

Accepted: 18 March, 2020

Published: 19 March, 2020

*Corresponding author: Ana Fernández-Araque, PhD, Department of Nursing, Professor at the Faculty of Health Sciences, University of Valladolid, Soria Campus, s/n 42004 Soria, Spain, Tel: 97512-9505; +34 975129514; Fax: 975129501 .

E-mail: anamaria.fernandez@uva.es, afa@enf.uva.es https://www.peertechz.com

\section{Check for updates}

\author{
Ana Fernández-Araque ${ }^{1,2 *}$, Mercedes Martínez-Delgado ${ }^{3}$, \\ Zoraida Verde-Rello ${ }^{2,4}$ and Verónica Velasco-González ${ }^{2,5}$ \\ ${ }^{1} \mathrm{PhD}$, Department of Nursing, Faculty of Health Sciences, University of Valladolid, Spain \\ ${ }^{2}$ Researcher in the Pharmacogenetics, Genetic Polymorphisms and Pharmacy epidemiology Research \\ Group, University of Valladolid, Spain \\ ${ }^{3} \mathrm{PhD}$, candidate in Non-Healing Wounds, Nurse at the Soria Correctional Facility, Department of \\ Anatomy and Radiology, Doctoral School, University of Valladolid, Spain \\ ${ }^{4} \mathrm{PhD}$, Department of Biochemistry, Faculty of Health Sciences, University of Valladolid, Spain \\ ${ }^{5} \mathrm{PhD}$, Department of Nursing, Faculty of Nursing, University of Valladolid, Spain
}

\section{Abstract}

The author presents the case of a 63-year-old man who was diagnosed with type 2 diabetes 15years ago (2004) and with ulcers in both feet with 12years of clinical course (which have required hospital admissions). The patient was diagnosed with moderate Charcot neuroarthropathy in 2009 using foot X-rays, receiving no specific treatment for this condition. The ulcers had been treated with multiple healing products, with poor results, for ten years.

The author presents the implementation of a care plan called DOMINATE for wound treatment and improved metabolic control of the concomitant condition. This case illustrates how important it is that health care providers consider an urgent need to establish a multidisciplinary care plan for the treatment of patients with nonhealing wounds so that these patients do not have to wait months or even years to be assessed by different specialists.

\section{Case study}

A 63-year-old male patient was diagnosed with type 2 diabetes 15 years ago (2004). An estimated $13.8 \%$ of the Spanish population has diabetes [1] and it is expected that the number of individuals with diabetes will reach $4.4 \%$ of the world population by 2030 . The patient presented with dyslipidemia, polyneuropathy and ulcers in both feet with 12 years of clinical course, which have required at least five hospital admissions to treat infections since the disease was diagnosed.

The patient was diagnosed with moderate Charcot neuroarthropathy in 2009 (Figure 1) and 2013 (Figure 2) using foot $\mathrm{X}$-rays, receiving no specific treatment for this condition.

\section{What we know}

Charcot neuroarthropathy has been associated with up to 24 different diseases. The reality is that diabetes is not the only cause of this condition. Any disease that causes sensory and autonomic neuropathy can cause these types of joint deformities [2]. The most common factors that lead to amputation include lower limb infections, gangrene, diabetic foot ulcers, and ischemia. Patients with a history of prior amputation, peripheral vascular disease, and neuropathy were more likely to require amputation [3].

For 11 years, the patient has had ulcers of varying depths and sizes in the metatarsophalangeal region of both feet. The patient presented with smaller ulcers on the right foot, in the 


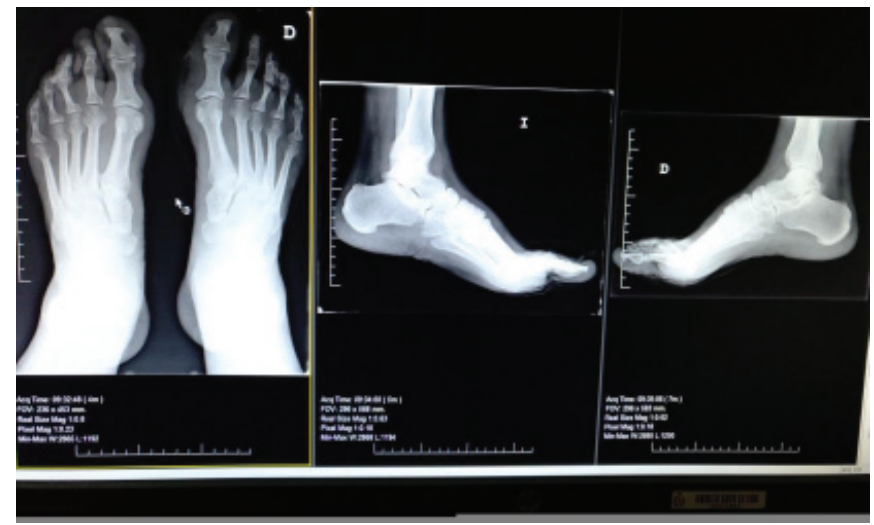

Figure 1: X-ray of both feet of 2009.

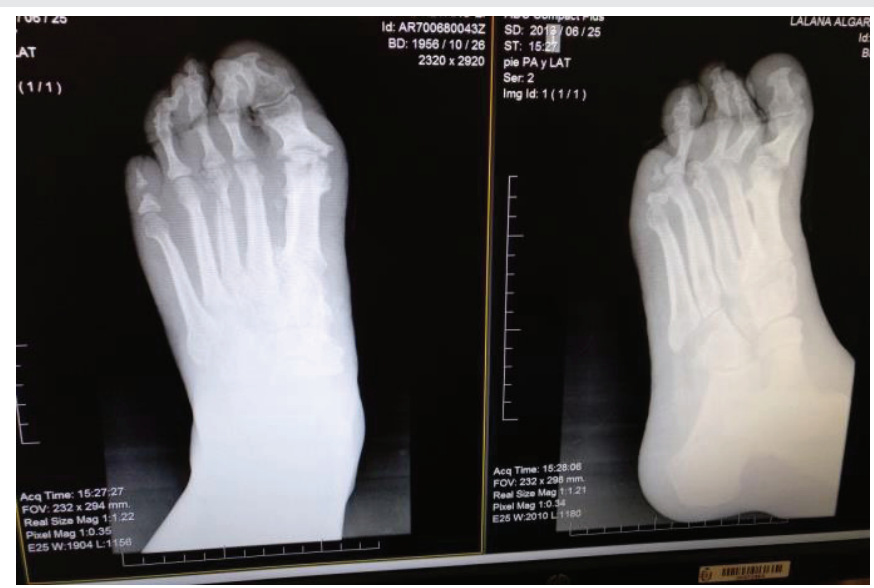

Figure 2: X-rays from the year of 2013

hallux metatarsophalangeal joint. The patient also presented with ulcers on the left foot, on the first and second toes, in the plantar metatarsophalangeal region. The ulcers had been treated with multiple healing products, with poor results, for ten years. The patient had been seen by the vascular surgeon to check for a moderate ischemia. The patient had not had an Ankle-Brachial Pressure Index (ABPI) test, but he had had a Doppler ultrasonography. The patient reported that, on several occasions, a supracondylar amputation had been suggested to him, but the patient rejected this on many other occasions [3]. Lower-extremity amputations are preceded by diabetic foot ulcers in up to $85 \%$ of cases. All of these data give us a notion of the loss in the quality of life of these patients and the economic impact that this condition has on the health system.

\section{Ongoing medical treatment on admission:}

- Glargine insulin: 28units at breakfast.

- Glusina insulin for hyperglycemia.

- $\quad$ Atorvastatin: $10 \mathrm{mg}$ every $24 \mathrm{~h}$.

- Acetylsalicylic acid: 100mg every $24 \mathrm{~h}$.

- Vitamin B1-B6-B12:1 every 24h.

Ongoing wound treatment on admission:
- $\quad$ Cleansing with regular soap.

- Honey for domestic use.

- $\quad$ Offloading with felt padding.

- Hyperoxygenated Fatty Acids (HOFA).

- Examination upon admission:

Upon admission to the health center, the patient presented with ulcers on both feet, with larger ulcers on the left foot $(3 \times 4 \mathrm{~cm}$ and $4 \times 2 \mathrm{~cm})$, and relatively good metabolic control with a glycosylated hemoglobin value of $7.4 \mathrm{mmol} / \mathrm{L}$. The left extremity showed edema with signs of chronicity, since granular edema appears in the root of the toes. The pedal and tibial right foot pulses were somewhat easily felt, unlike the pulses of the left foot.

- $\quad$ Blood pressure: $140 / 89$

- Glycosylated hemoglobin: $7.4 \mathrm{mmol} / \mathrm{L}$

- Cholesterol: $175 \mathrm{mg} / \mathrm{dL}$

- Weight: $76.8 \mathrm{~kg}$

- Height: $176 \mathrm{~cm}$

- $\quad$ BMI: 24 (normal weight)

- $\quad$ Right ABPI: $1.2 \mathrm{~cm}$; left ABPI: $1.3 \mathrm{~cm}$.

\section{Care plan}

A care plan was established following the acronym DOMINATE for wound treatment and improved metabolic control of the concomitant condition:

Debridement (D): Sharp debridement of periwound hyperkeratosis.

Offloading (0): Offloading of metatarsal heads, especially of the hallux, was carried out using $1 \mathrm{~cm}$-thick double felt padding, crutches, and orthopedic shoes with anterior offloading.

Moisture (M): Cadexomer iodine (Iodosorb $\rightarrow$ ) and calcium alginate dressings were applied for exudate control. Medications $(\mathrm{M})$ : No medication was prescribed that may hinder the healing process. Mental health: A relationship of trust was established between the patient and the nurse, so that the patient can trust the professional who treats him.

Infection (I): Wound culture was performed on two occasions due to increased exudate and lack of healing, revealing colonization by Staphylococcus aureus, which was treated with oral antibiotics given the lack of progress in healing. A probe-to-bone test was performed: the result was negative. Foot X-rays were also performed to check for possible osteomyelitis, which was discarded in principle (Figure 3).

Inflammation (I): Once we verified that the arterial compromise was moderate, in accordance with the vascular surgery report, we applied, with the due precautions, low- 
stretch multicomponent bandages with which the patient is without discomfort.

Nutrition (N): The patient was seen at the endocrinologist's office. A diabetic diet was prescribed, setting a goal of a glycosylated hemoglobin value of $7 \mathrm{mmol} / \mathrm{L}$.

Arterial insufficiency (A): Pedal and tibial pulses were found to be normal in the right foot, but weakened in the left foot. An ABPI test was performed, obtaining $1.2 \mathrm{~cm}$ in the right foot and $1.3 \mathrm{~cm}$ in the left foot, the latter being indicative of possible arterial calcification. The patient visited a surgeon, who requested Doppler ultrasonography, showing moderate arteriopathy. The left leg edema was eliminated and the patient showed less discomfort.

Technical advances (T): Low-stretch multicomponent bandages, used to reduce the edema of the left extremity, could be considered to belong to this section.

Edema (E): Control of the edema using low-stretch multicomponent bandages and postural measures, as well as a lower extremity exercise plan to facilitate venous return.

Education (E): The patient was trained with respect to his baseline disease process, diabetes, and his current foot ulcer condition, as well as their causes. The patient was also periodically reminded of the dietary and offloading measures to follow to have good control of his diabetes and his Charcot neuroarthropathy (Figures 3-5).

\section{Outcome}

After two months, the ulcer on the right foot was completely closed, but the ulcer on the left foot was not. The previously established plan was followed and a foot X-ray was requested. Subsequently, the patient visited the trauma specialist, who assessed the treatment of the Charcot neuroarthropathy and established a differential diagnosis with chronic osteomyelitis [4].

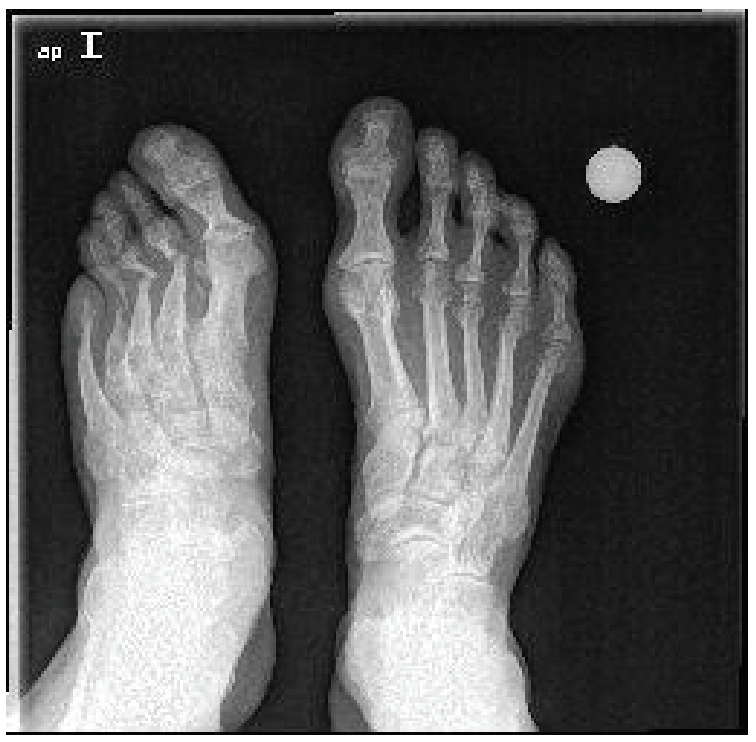

Figure 3: Right and left feet RX-AP of 2018.

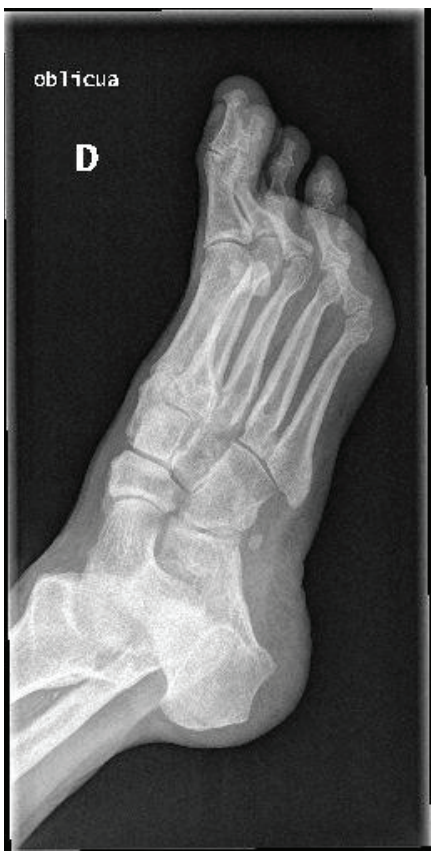

Figure 4: Oblique right foot of 2018.

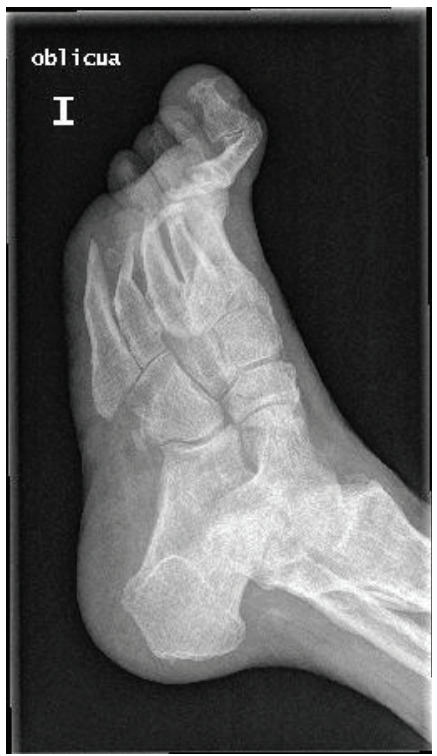

Figure 5: Oblique left foot of 2018

At the time of this evaluation, the patient had a $1 \times 1 \mathrm{~cm}$ plantar wound on the left foot, with abundant periwound hyperkeratosis, red wound bed with granulation tissue, thickened edges, occasionally macerated due to moderate to high levels of exudate. Despite wound care and the improvement in the underlying condition, complete closure of the ulcer was not achieved. The patient presented with a deformity of the sole of the foot, in the tarsometatarsal joint of the hallux [5], which was sometimes painful despite his sensory neuropathy.

The patient was informed about the X-ray results and was advised to rest as much as possible and to refrain from leaning on the left foot due to the possibility of bone fractures and complications greater than those already observed in the 
X-ray. The offloading of the foot was maximized by applying a $1.5 \mathrm{~cm}$ felt pad and by replacing the orthopedic shoe with another orthopedic shoe with more anterior offloading.

\section{Research is needed}

The patient has a diabetic neuropathy which had progressed poorly and resulted in severe Charcot neuroarthropathy. Bone deformities had led the patient to rest on the sole of the foot inadequately, which, in turn, caused non-healing diabetic foot ulcers, since the fundamental cause had not been resolved. Charcot neuroarthropathy may be scientifically explained by neurovascular causes, by which the increase of peripheral blood in the bones of the feet produces bone resorption, demineralization and osteopenia. Due to autonomic neuropathy, sympathetic vascular tone is lost, resulting in increased perfusion. The destruction of the autonomic fibers results in increased blood flow and bone hyperemia [6]. Diabetic foot patients should be treated simultaneously by professionals from multiple disciplines: endocrinologists, vascular surgeons, podiatrists, trauma specialists, and nurses, mainly. Different specialists treating the patient individually (i.e. without coordination) can prevent the correct resolution of the problems that a patient of this type may present with. This, in turn, implies much higher costs for the health system due to the prolonged duration of the care delivered and the corresponding costs in human and material resources.

Having diabetic foot units and referral non-healing wounds units is an essential need of any health system. These units would provide benefits in economic terms and in terms of patients' quality of life. The X-rays included are representative of the course of Charcot neuroarthropathy in a diabetic foot that has not been treated adequately due to the lack of a coordinated multidisciplinary team, thus causing harm to both the patient and the health system.

\section{Acknowledgments}

The authors would like to thank Dr. Lázaro (Head of the
Diabetic Foot Unit at the Complutense University of Madrid, Spain) and Dr. Barbero (Division of Diabetes Medicine, Gerencia de Asistencia Sanitaria, Soria, Spain) for their valuable comments on the manuscript. The authors thank Dr. Rabadán (Department of Laboratory Medicine, Santa Bárbara Hospital, Soria, Spain) for his help with interpreting the laboratory results.

\section{Author contributions}

A.F.A. and M.M.D. wrote the manuscript. V.V.R. interpreted the laboratory results. V.V.G. critically reviewed the manuscript. M.M.D. is the guarantor of this work and, as such, had full access to all the data in the study and takes responsibility for the integrity of the data and the accuracy of the data analysis.

\section{References}

1. Edmonds M, Lázaro-Martínez JL, Alfayate-García JM, Martini J, Petit JM, et al. (2017) Sucrose octasulfate dressing versus control dressing in patients with neuroischaemic diabetic foot ulcers (Explorer): an international, multicentre double-blind, randomised, controlled trial. Lancet Diabetes Endocrinol 6: 186196. Link: https://bit.ly/395EmCd

2. Jordan W (1936) Neuritic manifestations in diabetes mellitus. JAMA Interna Medicine 57: 307-366. Link: https://bit.ly/395WsE8

3. Aschenbrenner D (2017) Diabetes Drug Receives Boxed Warning for Increased Risk of Leg and Foot Amputations. Am J Nurs 117: 22-23. Link: https://bit.ly/2UkjX6M

4. Lee RC, Frykberg RG, Armstrong DG, Boulton AJ, Edmonds M, et al. (2011) The Charcot Foot in Diabetes. J Am Podiatr Med Assoc 101: 437-446. Link: https://bit.ly/38V8iR7

5. Milne TE, Rogers JR, Kinnear EM, Martin HV, Lazzarini PA, et al. (2013) Developing an evidence-based clinical pathway for the assessment, diagnosis and management of acute charcot neuro-arthropathy: a systematic review. J Foot Ankle Res 6: 30. Link: https://bit.ly/2TWOuJ0

6. Molines-Barroso RJ, Lázaro-Martínez JL, Beneit-Montesinos JV, GarcíaMorales E, Álvaro-Afonso FJ, et al. (2019) Predictors of Diabetic foot reulceration beneath the Hallux. J Diabetes Res 2019: 9038171. Link: https://bit.ly/2IX45Ss

Discover a bigger Impact and Visibility of your article publication with

Peertechz Publications

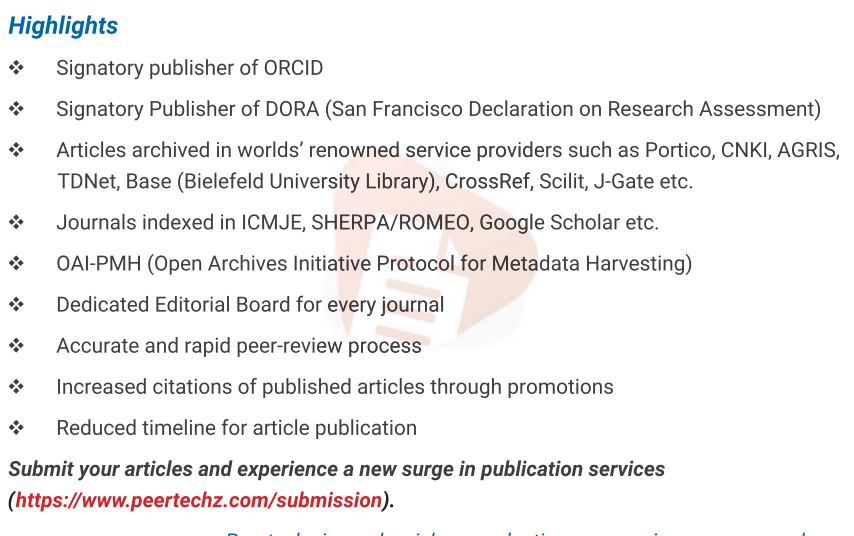

Peertechz journals wishes everlasting success in your every endeavours.

Copyright: ( $) 2020$ Araque AF, et al. This is an open-access article distributed under the terms of the Creative Commons Attribution License, which permits unrestricted use distribution, and reproduction in any medium, provided the original author and source are credited. 